\title{
The Second Hyper-Zagreb Coindex of Chemical Graphs and Some Applications
}

\author{
Ahmed Ayache $\mathbb{D}^{1},{ }^{1}$ Abdu Alameri $\mathbb{D}{ }^{2}$ Mohammed Alsharafi $\mathbb{D}^{2},{ }^{3}$ and Hanan Ahmed $\mathbb{D}^{4}$ \\ ${ }^{1}$ Department of Mathematics, Faculty of Science, University of Bahrain, Zallaq, Bahrain \\ ${ }^{2}$ Department of Biomedical Engineering, Faculty of Engineering, University of Science and Technology, Taiz, Yemen \\ ${ }^{3}$ Graduate School of Natural and Applied Sciences, Yildiz Technical University, Istanbul, Turkey \\ ${ }^{4}$ Department of Mathematics, Yuvaraja's College, University of Mysore, Mysuru, India \\ Correspondence should be addressed to Abdu Alameri; a.alameri2222@gmail.com
}

Received 10 September 2021; Accepted 21 October 2021; Published 15 November 2021

Academic Editor: Syed Ahtsham Haq Bokhary

Copyright (c) 2021 Ahmed Ayache et al. This is an open access article distributed under the Creative Commons Attribution License, which permits unrestricted use, distribution, and reproduction in any medium, provided the original work is properly cited.

\begin{abstract}
The second hyper-Zagreb coindex is an efficient topological index that enables us to describe a molecule from its molecular graph. In this current study, we shall evaluate the second hyper-Zagreb coindex of some chemical graphs. In this study, we compute the value of the second hyper-Zagreb coindex of some chemical graph structures such as sildenafil, aspirin, and nicotine. We also present explicit formulas of the second hyper-Zagreb coindex of any graph that results from some interesting graphical operations such as tensor product, Cartesian product, composition, and strong product, and apply them on a $q$-multiwalled nanotorus.
\end{abstract}

\section{Introduction}

A graph can be identified by a corresponding numerical value, a sequence of numbers, or a special polynomial or a matrix. Special attention is directed to chemical graphs which constitute a wonderful topic in graph theory because of the abundance of applications in chemistry or in medical science $[1,2]$. Topological index and coindex are invariant under graph automorphism. The computation of these numerical quantities is useful and well-proven in medical information of new drugs without resorting to chemical experiments $[3,4]$. All graphs in this study are finite and simple, let $G$ be a finite simple graph on $V(G)=n$, vertices, and $E(G)=m$, edges, and the degree of a vertex $v$ is the number of edges event to $v$, denoted by $\delta_{G}(v)$. The complement of $G$, denoted by $\bar{G}$, is a simple graph on the same set of vertices $V(G)$, in which two vertices $u$ and $v$ are adjacent by an edge $u v$, if and only if they are not adjacent in $G$. Hence, $u v \in E(\bar{G})$ if and only if $u v \notin E(G)$. Obviously, we have $E(G) \bigcup E(\bar{G})=E\left(K_{n}\right)$, so $\bar{m}=E(\bar{G})=\left(\begin{array}{l}n \\ 2\end{array}\right)-m$, and the degree of a vertex $u$ in $\bar{G}$ is given by

$$
\delta_{\bar{G}}(u)=n-1-\delta_{G}(u) .
$$

Gutman and Trinajestić [5] introduced the first and second Zagreb indices as follows:

$$
\begin{aligned}
& M_{1}(G)=\sum_{v \in V(G)} \delta_{G}^{2}(v)=\sum_{u v \in E(G)}\left[\delta_{G}(u)+\delta_{G}(v)\right], \\
& M_{2}(G)=\sum_{u v \in E(G)} \delta_{G}(u) \delta_{G}(v) .
\end{aligned}
$$

In 2008, Došlić defined Zagreb coindices [6], which are given as follows:

$$
\begin{aligned}
& \bar{M}_{1}(G)=\sum_{u v \notin E(G)}\left[\delta_{G}(u) \delta_{G}(v)\right], \\
& \bar{M}_{2}(G)=\sum_{u v \notin E(G)}\left[\delta_{G}(u) \delta_{G}(v)\right] .
\end{aligned}
$$

Later in 2010, Ashrafi et al. have established the following nice formulas for the precise relationship between the first and second Zagreb indices and their coindices [7]: 


$$
\begin{aligned}
& \bar{M}_{1}(G)=2 m(n-1)-M_{1}(G), \\
& \bar{M}_{2}(G)=2 m^{2}-\frac{1}{2} M_{1}(G)-M_{2}(G) .
\end{aligned}
$$

In 2013, Shirdel et al. [8] introduced degree-based Zagreb indices named hyper-Zagreb index which is defined as

$$
H M(G)=\sum_{u v \in E(G)}\left(\delta_{G}(u)+\delta_{G}(v)\right)^{2} .
$$

In 2013, Ranjini et al. introduced and defined the third Zagreb index of a graph as [9]

$$
\operatorname{Re}_{3}(G)=\sum_{u v \in E(G)} \delta_{G}(u) \delta_{G}(v)\left[\delta_{G}(u)+\delta_{G}(v)\right] .
$$

Furtula and Gutman in 2015 introduced the forgotten index (F-index) [10], which is defined as

$$
F(G)=\sum_{v \in V(G)} \delta_{G}^{3}(v)=\sum_{u v \in E(G)}\left[\delta_{G}^{2}(u)+\delta_{G}^{2}(v)\right] .
$$

In 2016, De et al. introduced forgotten coindex as follows:

$$
\begin{aligned}
\bar{F}(G) & =\sum_{u v \notin E(G)}\left[\delta_{G}^{2}(u)+\delta_{G}^{2}(v)\right] \\
& =(n-1) M_{1}(G)-F(G) .
\end{aligned}
$$

In 2016, Veylaki et al. [11] introduced hyper-Zagreb coindex as follows:

$$
\overline{H M}(G)=\sum_{u v \notin E(G)}\left(\delta_{G}(u)+\delta_{G}(v)\right)^{2} .
$$

In 2016, Wei et al. [12] defined new version of Zagreb topological indices. It is called the hyper-Zagreb index that is defined as above. Then, the second hyper-Zagreb index of a graph $G$ is defined as the sum of the weights $\left(\delta_{G}(u) \delta_{G}(v)\right)^{2}$ and is equal to

$$
H M_{2}(G)=\sum_{u v \in E(G)}\left(\delta_{G}(u) \delta_{G}(v)\right)^{2} .
$$

In 2020, Alameri et al. [13, 14] defined a new degreebased of Zagreb indices named Y-index and Y-coindex as

$$
\begin{aligned}
& Y(G)=\sum_{u v \in E(G)}\left[\delta_{G}^{3}(u)+\delta_{G}{ }^{3}(v)\right], \\
& \bar{Y}(G)=\sum_{u v \notin E(G)}\left[\delta_{G}^{3}(u)+\delta_{G}^{3}(v)\right],
\end{aligned}
$$

where

$$
\bar{Y}(G)=(n-1) F(G)-Y(G) .
$$

Here, we define a new version of Zagreb topological indices, based on the hyper-Zagreb index that is defined as above. It is called the second hyper-Zagreb index of a graph $G$ and defined as the sum of the weights $\left(\delta_{G}(u) \delta_{G}(v)\right)^{2}$, such that $u v \notin E(G)$ and is equal to

$$
\overline{H M_{2}}(G)=\sum_{u v \notin E(G)}\left(\delta_{G}(u) \delta_{G}(v)\right)^{2} .
$$

Eventhough, there are several research reports contributing to the computation of topological indices of chemical graphs. However, the studies on the computation of topological coindices of octane isomers are very limited. This study focused on one of the important topological coindices named the second hyper-Zagreb coindex. Some chemical graphs were obtained by this parameter. Moreover, the second hyper-Zagreb coindex of graph operations was computed and gave some of their applications such as a $q$ multiwalled nanotorus.

\section{Preliminaries}

This section is devoted to some preparatory results that will play a prominent role in our study.

Definition 2.1 (see $[15,16]$ ). Suppose that $G_{1}$ and $G_{2}$ are two connected graphs, then

(i) The tensor product $G_{1} \otimes G_{2}$ of $G_{1}$ and $G_{2}$ is the graph with $V\left(G_{1} \otimes G_{2}\right)=V\left(G_{1}\right) \times V\left(G_{2}\right)$, and $E\left(G_{1} \otimes G_{2}\right)=\left\{\left(u_{1}, u_{2}\right)\left(v_{1}, v_{2}\right) \mid u_{1} v_{1} \in E\left(G_{1}\right)\right.$, $\left.u_{2} v_{2} \in E\left(G_{2}\right)\right\}$.

(ii) The Cartesian product $G_{1} \times G_{2}$ of $G_{1}$ and $G_{2}$ has the vertex set $V\left(G_{1} \times G_{2}\right)=V\left(G_{1}\right) \times V\left(G_{2}\right)$, and $(a, x)(b, y)$ is an edge of $G_{1} \times G_{2}$ if $a=b$ and $x y \in E\left(G_{2}\right)$ or $a b \in E\left(G_{1}\right)$ and $x=y$.

(iii) The composition $G_{1}\left[G_{2}\right]$ of $G_{1}$ and $G_{2}$ with disjoint vertex sets $V\left(G_{1}\right)$ and $V\left(G_{2}\right)$ and edge sets $E\left(G_{1}\right)$ and $E\left(G_{2}\right)$ is the graph with vertex set $V\left(G_{1}\right) \times$ $V\left(G_{2}\right)$ and any two vertices $u=\left(u_{1}, v_{1}\right)$ is adjacent with $v=\left(u_{2}, v_{2}\right)$ whenever $\left(u_{1}\right.$ is adjacent with $\left.u_{2}\right)$ or $\left(u_{1}=u_{2}\right.$ and $v_{1}$ is adjacent with $\left.v_{2}\right)$.

(iv) The strong product $G_{1} * G_{2}$ of $G_{1}$ and $G_{2}$ is a graph with $V\left(G_{1} * G_{2}\right)=V\left(G_{1}\right) \times V\left(G_{2}\right)$, and any two vertices $\left(u_{1}, v_{1}\right)$ and $\left(u_{2}, v_{2}\right)$ are adjacent if and only if $\left\{u_{1}=u_{2} \in V\left(G_{1}\right) \quad\right.$ and $\left.v_{1} v_{2} \in E\left(G_{2}\right)\right\} \quad$ or $\left\{v_{1}=v_{2} \in V\left(G_{2}\right)\right.$ and $\left.u_{1} u_{2} \in E\left(G_{1}\right)\right\}$.

Lemma 2 (see $[17,18]$ ). Let $G_{1}$ and $G_{2}$ be graphs with $\left|V\left(G_{1}\right)\right|=n_{1},\left|V\left(G_{2}\right)\right|=n_{2},\left|E\left(G_{1}\right)\right|=m_{1}$, and $\left|E\left(G_{2}\right)\right|=$ $m_{2}$. Then,

(i) $\left|V\left(G_{1} \otimes G_{2}\right)\right|=\left|V\left(G_{1} \times G_{2}\right)\right|=\left|V\left(G_{1}\left[G_{2}\right]\right)\right|=$

$\left|V\left(G_{1} * G_{2}\right)\right|=n_{1} n_{2}$

(ii) $E\left(G_{1} \otimes G_{2}\right) \mid=2 m_{1} m_{2}$

(iii) $\left|E\left(G_{1} \times G_{2}\right)\right|=m_{1} n_{2}+n_{1} m_{2}$

(iv) $\left|E\left(G_{1}\left[G_{2}\right]\right)\right|=m_{1} n_{2}^{2}+m_{2} n_{1}$

(v) $\left|E\left(G_{1} * G_{2}\right)\right|=m_{1} n_{2}+n_{1} m_{2}+2 m_{1} m_{2}$

(vi) $\delta_{G_{1} \otimes G_{2}}(u, v)=\delta_{G_{1}}(u) \delta_{G_{2}}(v)$
(a) $\delta_{G_{1} \times G_{2}}(u, v)=\delta_{G_{1}}(u)+\delta_{G_{2}}(v)$
(b) $\delta_{G_{1}\left[G_{2}\right]}(u, v)=n_{2} \delta_{G_{1}}(u)+\delta_{G_{2}}(v)$
(c) $\delta_{G_{1} * G_{2}}(u, v)=\delta_{G_{1}}(u)+\delta_{G_{2}}$ $(v)+\delta_{G_{1}}(u) \delta_{G_{2}}(v)$ 
Lemma 2.3 (see $[17,18]$ ). Let $G_{1}, G_{2}$ be two graphs with $n_{1}, n_{2}$ vertices and $m_{1}, m_{2}$ edges, respectively, then.

(i) $M_{1}\left(G_{1} \otimes G_{2}\right)=M_{1}\left(G_{1}\right) M_{1}\left(G_{2}\right)$

(ii) $M_{1}\left(G_{1} \times G_{2}\right)=n_{2} M_{1}\left(G_{1}\right)+n_{1} M_{1}\left(G_{2}\right)+8 m_{1} m_{2}$

(iii) $M_{1}\left(G_{1}\left[G_{2}\right]\right)=n_{2}^{3} M_{1}\left(G_{1}\right)+n_{1} M_{1}\left(G_{2}\right)+$ $8 n_{2} m_{2} m_{1}$

(iv) $M_{1}\left(G_{1} * G_{2}\right)=\left(n_{2}+6 m_{2}\right) M_{1}\left(G_{1}\right)+8 m_{2} m_{1}+$ $\left(6 m_{1}+n_{1}\right) M_{1}\left(G_{2}\right)+2 M_{1}\left(G_{1}\right) M_{1}\left(G_{2}\right)$

Lemma 2.4 (see $[17,18]$ ). Let $G_{1}, G_{2}$ be two simple graphs with $n_{1}, n_{2}$ vertices and $m_{1}, m_{2}$ edges, respectively, then

(i) $Y\left(G_{1} \otimes G_{2}\right)=Y\left(G_{1}\right) Y\left(G_{2}\right)$

(ii) $Y\left(G_{1} \times G_{2}\right)=n_{2} Y\left(G_{1}\right)+n_{1} Y\left(G_{2}\right)+8 m_{1} F$

$\left(G_{2}\right)+8 m_{2} F\left(G_{1}\right)+6 M_{1}\left(G_{1}\right) M_{1}\left(G_{2}\right)$

(iii) $Y\left(G_{1}\left[G_{2}\right]\right)=n_{2}{ }^{5} Y\left(G_{1}\right)+\quad n_{1} Y\left(G_{2}\right)+8 n_{2}{ }^{3} m_{2} F$ $\left(G_{1}\right)+8 n_{2} m_{1} F\left(G_{2}\right)+6 n_{2}{ }^{2} M_{1}\left(G_{1}\right) M_{1}\left(G_{2}\right)$

(iv) $Y\left(G_{1} * G_{2}\right)=Y\left(G_{1}\right)\left[4 F\left(G_{2}\right)+6 M_{1}\left(G_{2}\right)+8 m_{2}+\right.$ $\left.n_{2}\right]+4 F\left(G_{1}\right)\left[3 M_{1}\left(G_{2}\right)+2 m_{2}\right]+Y\left(G_{2}\right)\left[4 F\left(G_{1}\right)+\right.$ $\left.6 M_{1}\left(G_{1}\right)+8 m_{1}+n_{1}\right]+4 F\left(G_{2}\right)\left[3 M_{1}\left(G_{1}\right)+2 m_{1}\right]+$ $Y\left(G_{1}\right) Y\left(G_{2}\right)+12 F\left(G_{1}\right) F\left(G_{2}\right)+6 M_{1}\left(G_{1}\right) M_{1}\left(G_{2}\right)$

Lemma 2.5 (see $[17,18]$ ). Let $G_{1}, G_{2}$ be two simple graphs with $n_{1}, n_{2}$ vertices and $m_{1}, m_{2}$ edges, respectively, then

(i) $H M_{2}\left(G_{1} \otimes G_{2}\right)=2 H M_{2}\left(G_{1}\right) H M_{2}\left(G_{2}\right)$

(ii) $\mathrm{HM}_{2}\left(G_{1} \times G_{2}\right)=n_{2} H M_{2}\left(G_{1}\right)+n_{1} H M_{2}\left(G_{2}\right)+3 F$ $\left(G_{1}\right) M_{1}\left(G_{2}\right)+3 F\left(G_{2}\right) M_{1}\left(G_{1}\right)+m_{1}\left[Y\left(G_{2}\right)+\right.$ $\left.4 \operatorname{ReZ} G_{3}\left(G_{2}\right)\right]+m_{2}\left[Y\left(G_{1}\right)+4 \operatorname{ReZ} G_{3}\left(G_{1}\right)\right]+4 M_{1}$ $\left(G_{1}\right) M_{2}\left(G_{2}\right)+4 M_{1}\left(G_{2}\right) M_{2}\left(G_{1}\right)$

(iii) $\mathrm{HM}_{2}\left(G_{1}\left[G_{2}\right]\right)=n_{2}{ }^{6} \mathrm{HM}_{2}\left(G_{1}\right)+n_{1} \mathrm{HM}_{2}\left(G_{2}\right)+$ $n_{2}{ }^{4} m_{2}\left[Y\left(G_{1}\right)+4 \operatorname{Re} Z G_{3}\left(G_{1}\right)\right]+4 n_{2} m_{1}$ $\operatorname{Re} Z G_{3}\left(G_{2}\right)+3 n_{2}{ }^{2} F\left(G_{1}\right) M_{1}\left(G_{2}\right)+n_{2}{ }^{2} M_{1}\left(G_{1}\right)$

$$
\left[F\left(G_{2}\right)+4 M_{2}\left(G_{2}\right)\right]+m_{1} M_{1}^{2}\left(G_{2}\right)+4 n_{2} m_{2}
$$$$
\left[4 n_{2} m_{2} M_{2}\left(G_{1}\right)+M_{1}\left(G_{1}\right) M_{1}\left(G_{2}\right)\right]
$$

(iv) $H M_{2}\left(G_{1} * G_{2}\right)=H M_{2}\left(G_{2}\right) \quad\left[n_{1}+10 m_{1}+10 m_{1}\right.$

$\left.\left(G_{1}\right)+8 m_{2}\left(G_{1}\right)+6 F\left(G_{1}\right)+4 R e Z G_{3}\left(G_{1}\right)+Y\left(G_{1}\right)\right]$ $+H M_{2}\left(G_{2}\right)\left[n_{2}+10 m_{2}+10 m_{1}\left(G_{2}\right)+8 m_{2}\left(G_{2}\right)+\right.$ $\left.6 F\left(G_{2}\right)+4 R e Z G_{3}\left(G_{2}\right)+Y\left(G_{2}\right)\right]+Y\left(G_{2}\right)\left[m_{1}+\right.$ $\left.2 M_{1}\left(G_{1}\right)+4 M_{2}\left(G_{1}\right)+F\left(G_{1}\right)+2 R e Z G_{3} \quad\left(G_{1}\right)\right] Y$ $\left(G_{1}\right)\left[m_{2}+2 M_{1}\left(G_{2}\right)+4 M_{2}\left(G_{2}\right)+F\left(G_{2}\right)+\right.$ $\left.2 \operatorname{ReZG} G_{3}\left(G_{2}\right)\right]+4 \operatorname{ReZG} G_{3}\left(G_{2}\right) \quad\left[m_{1}+2 M_{1} \quad\left(G_{1}\right)\right.$ $\left.+2 M_{2} \quad\left(G_{1}\right)+2 F\left(G_{1}\right)\right]+4 \operatorname{ReZG}_{3}\left(G_{1}\right) \quad\left[m_{2}+\right.$ $\left.2 M_{1}\left(G_{2}\right)+2 M_{2}\left(G_{2}\right)+2 F\left(G_{2}\right)\right]+F\left(G_{2}\right)\left[3 M_{1}\right.$ $\left.\left(G_{1}\right)+8 M_{2}\left(G_{1}\right)\right]+4 M_{1}\left(G_{1}\right) M_{2}\left(G_{2}\right)+4 M_{1}\left(G_{2}\right)$ $M_{2}\left(G_{1}\right)+2 H M_{2}\left(G_{1}\right) H M_{2}\left(G_{2}\right)+5 F\left(G_{1}\right) F\left(G_{2}\right)+$ $6 \operatorname{ReZG}_{3}\left(G_{1}\right) \operatorname{ReZG} G_{3}\left(G_{2}\right)$

\section{Main Results}

In the following section, we study the second hyper-Zagreb coindex of some chemical graph structures, exactly sildenafil, aspirin, and nicotine.

Proposition 3.1. Let $G$ be a graph with $n$ vertices and $m$ edges. Then,

$$
\overline{H M}(G)=(n-2) M_{1}(G)+4 m^{2}-H M(G) .
$$

Proof. For the proof (Theorem 3.2), we refer to [10].

Proposition 3.2. Let $G$ be a graph with $n$ vertices and $m$ edges. Then,

$$
\overline{H M_{2}}(G)=\frac{1}{2} M_{1}^{2}(G)-\frac{1}{2} Y(G)-H M_{2}(G) .
$$

Proof. By definition of the second hyper-Zagreb coindex and using a similar method, as above in Proposition 3.1, then

$$
\begin{aligned}
H M_{2}(G)+\overline{H M_{2}}(G) & =\left[\sum_{u v \in E(G)}+\sum_{u v \notin E(G)}\right]\left[\delta_{G}(u) \delta_{G}(v)\right]^{2} \\
& =\frac{1}{2}\left[\sum_{u \in V(G)} \sum_{v \in V(G)}\left[\delta_{G}(u) \delta_{G}(v)\right]^{2}-\sum_{v \in V(G)}\left[\delta_{G}(v) \delta_{G}(v)\right]^{2}\right] \\
& =\frac{1}{2}\left[M_{1}^{2}(G)-Y(G)\right] .
\end{aligned}
$$

Sildenafil $\left(\mathrm{C}_{22} \mathrm{H}_{30} \mathrm{~N}_{6} \mathrm{O}_{4} \mathrm{~S}\right)$ is a drug used for pulmonary arterial hypertension. It is taken by mouth or injection into a vein (Figure 1) [19].

Proposition 3.3. The second hyper-Zagreb coindex of sildenafil.

From the graph structure of sildenafil (Figure 1), it is easy to obtain the dataset in Tables 1 and 2 .
By Table 1 and definitions of the first Zagreb index and the Y-index, we have

$$
\begin{aligned}
M_{1}(\text { sildenafil }) & =(7)(1)+(14)(4)+(11)(9)+(1)(16)=178, \\
Y(\text { sildenafil }) & =(7)(1)+(14)(16)+(11)(81)+(1)(256)=1378 .
\end{aligned}
$$

Also, by Table 2 and definition of the second hyperZagreb index, we have 


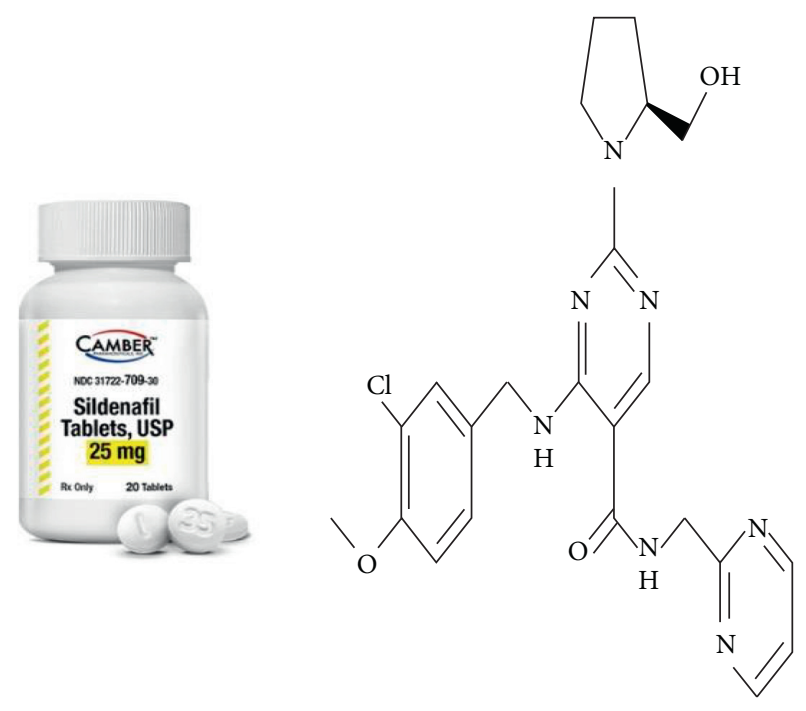

FIGURE 1: Graph structure of sildenafil.

TABle 1: Atoms dataset of the graph structure of sildenafil.

\begin{tabular}{lcccc}
\hline No. of atoms & 7 & 14 & 11 & 1 \\
\hline$\delta v$ & 1 & 2 & 3 & 4 \\
$\delta^{2} v$ & 1 & 4 & 9 & 16 \\
$\delta^{4} v$ & 1 & 16 & 81 & 256 \\
\hline
\end{tabular}

TABLE 2: Links dataset of the graph structure of sildenafil.

\begin{tabular}{lcccccc}
\hline No. of links & 2 & 3 & 7 & 16 & 6 & 2 \\
\hline$\delta^{2} u \delta^{2} v$ & 4 & 9 & 16 & 36 & 81 & 144 \\
\hline
\end{tabular}

$$
\begin{aligned}
H M_{2}(\text { sildenafil })= & (2)(4)+(3)(9)+(7)(16)+(16)(36) \\
& +(6)(81)+(2)(144)=1497
\end{aligned}
$$

Using Proposition 3.2, we have

$$
\overline{\mathrm{HM}_{2}} \text { ( sildenafil) }=13656 \text {. }
$$

Aspirin $\left(\mathrm{C}_{9} \mathrm{H}_{8} \mathrm{O}_{4}\right)$ is known as acetylsalicylic acid (ASA). Aspirin has many medicinal uses as it is a drug that is used to reduce fever or inflammation, also given after a heart attack to reduce the risk of death. Aspirin is also used as a nonsteroidal anti-inflammatory drug because it has an antiplatelet effect by inhibiting its normal functioning. Also, a lot of evidence indicates that aspirin is considered a chemical agent that may limit and reduce the incidence of general cancers (Figure 2) $[20,21]$.

Proposition 3.4. The second hyper-Zagreb coindex of aspirin.

From the graph structure of aspirin (Figure 2), it is easy to obtain the dataset in Tables 2 and 3.

Also, by Table 4 and definition of the second hyperZagreb index, we have

$$
H M_{2}(\text { aspirin })=(4)(9)+(3)(16)+(4)(36)+(2)(81)=390 .
$$
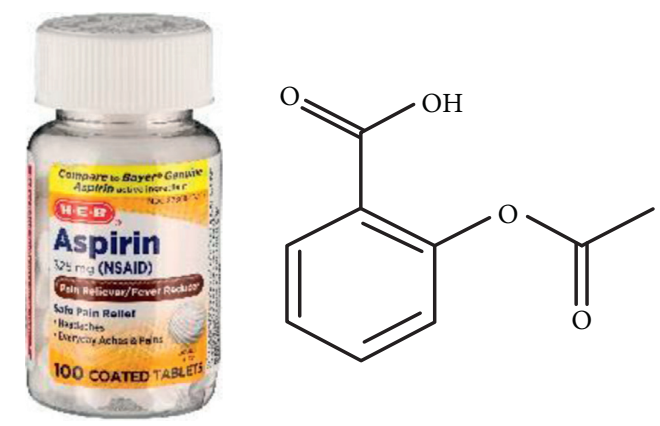

Figure 2: Graph structure of aspirin.

TABLE 3: Atoms dataset of the graph structure of aspirin.

\begin{tabular}{llcc}
\hline No. of atoms & 4 & 5 & 4 \\
\hline$\delta v$ & 1 & 2 & 3 \\
$\delta^{2} v$ & 1 & 4 & 9 \\
$\delta^{4} v$ & 1 & 16 & 81 \\
\hline
\end{tabular}

TABLE 4: Links dataset of the graph structure of aspirin.

\begin{tabular}{lcccc}
\hline No. of links & 4 & 3 & 4 & 2 \\
\hline$\delta^{2} u \delta^{2} v$ & 9 & 16 & 36 & 81 \\
\hline
\end{tabular}

Using Proposition 3.2, we have

$$
\left.\overline{H M_{2}} \text { (aspirin }\right)=1206 .
$$

Nicotine $\left(\mathrm{C}_{10} \mathrm{H}_{14} \mathrm{~N}_{2}\right)$ is an alkaloid that is widely used as an anxiolytic. Nicotine is used as a drug to quit smoking, and if it is not used well, it can lead to addiction. Many types of research conducted on animals indicate that some inhibitors found in tobacco smoke, such as monoamine oxidase, may enhance some of the addictive properties of nicotine (Figure 3) [21, 22]. Any unexplained terminology is standard, typically as in [22-24].

By Table 3 and definitions of the first Zagreb index and the Y-index, we have

$$
\begin{aligned}
M_{1}(\text { aspirin }) & =(4)(1)+(5)(4)+(4)(9)=60, \\
Y(\text { aspirin }) & =(4)(1)+(5)(16)+(4)(81)=408
\end{aligned}
$$

Proposition 3.5. The second hyper-Zagreb coindex of nicotine.

From the graph structure of nicotine (Figure 3), it is easy to obtain the dataset in Tables 5 and 6 .

By Table 5 and definitions of the first Zagreb index and the Y-index, we have

$$
\begin{aligned}
M_{1}(\text { nicotine }) & =(1)(1)+(8)(4)+(3)(9)=60, \\
Y(\text { nicotine }) & =(1)(1)+(8)(16)+(3)(81)=372 .
\end{aligned}
$$

Also, by Table 6 and definition of the second hyperZagreb index, we have 


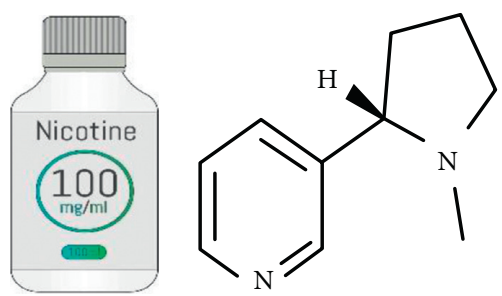

FIgURE 3: Graph structure of nicotine.

TABLE 5: Atoms dataset of the graph structure of nicotine.

\begin{tabular}{llcc}
\hline No. of atoms & 1 & 8 & 3 \\
\hline$\delta v$ & 1 & 2 & 3 \\
$\delta^{2} v$ & 1 & 4 & 9 \\
$\delta^{4} v$ & 1 & 16 & 81 \\
\hline
\end{tabular}

TABLE 6: Links dataset of the graph structure of nicotine.

\begin{tabular}{llccc}
\hline No. of links & 1 & 6 & 4 & 2 \\
\hline$\delta^{2} u \delta^{2} v$ & 9 & 16 & 36 & 81 \\
\hline
\end{tabular}

$$
\begin{aligned}
H M_{2}(\text { nicotine })= & (1)(9)+(6)(16)+(4)(36) \\
& +(2)(81)=483 .
\end{aligned}
$$

Using Proposition 3.2, we have

$$
\overline{H M_{2}}(\text { nicotine })=1131 \text {. }
$$

\section{Applications}

In the following section, we provide the exact value of the second hyper-Zagreb coindex of graphs that are arisen from mathematical operations such as the tensor product $G_{1} \otimes G_{2}$, the Cartesian product $G_{1} \times G_{2}$, the composition
$G_{1}\left[G_{2}\right]$, and the strong product $G_{1} * G_{2}$. Also, we apply this coindex on a $q$-multiwalled nanotorus.

Theorem 4.1. The second hyper-Zagreb coindex of $G_{1} \times G_{2}$ is given by

$$
\begin{aligned}
\overline{H M_{2}}\left(G_{1} \times G_{2}\right)= & \frac{1}{2}\left[n_{2} M_{1}\left(G_{1}\right)+n_{1} M_{1}\left(G_{2}\right)+8 m_{1} m_{2}\right]^{2} \\
& -\frac{1}{2}\left[n_{2} Y\left(G_{1}\right)+n_{1} Y\left(G_{2}\right)+8 m_{1} F\left(G_{2}\right)\right. \\
& \left.+8 m_{2} F\left(G_{1}\right)+6 M_{1}\left(G_{1}\right) M_{1}\left(G_{2}\right)\right] \\
& -\left[n_{2} H M_{2}\left(G_{1}\right)+n_{1} H M_{2}\left(G_{2}\right)\right. \\
& +3 F\left(G_{1}\right) M_{1}\left(G_{2}\right)+3 F\left(G_{2}\right) M_{1}\left(G_{1}\right) \\
& +m_{1}\left[Y\left(G_{2}\right)+4 \operatorname{Re} Z G_{3}\left(G_{2}\right)\right] \\
& +m_{2}\left[Y\left(G_{1}\right)+4 \operatorname{Re} Z G_{3}\left(G_{1}\right)\right]+4 M_{1} \\
& \left.\cdot\left(G_{1}\right) M_{2}\left(G_{2}\right)+4 M_{1}\left(G_{2}\right) M_{2}\left(G_{1}\right)\right] .
\end{aligned}
$$

Proof. We have $\overline{H M_{2}}(G)=1 / 2 M_{1}^{2}(G)-1 / 2 Y(G)-H$ $M_{2}(G)$, given in Proposition 3.2, and by replacing each $G$ by $\mathrm{G}_{1} \times \mathrm{G}_{2}$, which yields $\overline{H M_{2}}\left(G_{1} \times G_{2}\right)=1 / 2 M_{1}{ }^{2}\left(G_{1} \times G_{2}\right)$ $-1 / 2 Y\left(G_{1} \times G_{2}\right)-H M_{2}\left(G_{1} \times G_{2}\right)$, and by using (Lemma 2.2-Lemma 2.4), we obtain the required.

All proofs in Theorems $4.2-4.4$ are given as Theorem 4.1 .

Theorem 4.2. The second hyper-Zagreb coindex of $G_{1} * G_{2}$ is given by

$$
\begin{aligned}
\overline{H M}_{2}\left(G_{1} * G_{2}\right)= & \frac{1}{2}\left[\left(n_{2}+6 m_{2}\right) M_{1}\left(G_{1}\right)+8 m_{2} m_{1}\left(6 m_{1}+n_{1}\right) M_{1}\left(G_{2}\right)+2 M_{1}\left(G_{1}\right) M_{1}\left(G_{2}\right)\right]^{2} \\
& -\frac{1}{2}\left[Y\left(G_{1}\right)\left[4 F\left(G_{2}\right)+6 M_{1}\left(G_{2}\right)+8 m_{2}+n_{2}\right]+4 F\left(G_{1}\right)\left[3 M_{1}\left(G_{2}\right)+2 m_{2}\right]\right. \\
+ & Y\left(G_{2}\right)\left[4 F\left(G_{1}\right)+6 M_{1}\left(G_{1}\right)+8 m_{1}+n_{1}\right]+4 F\left(G_{2}\right)\left[3 M_{1}\left(G_{1}\right)+2 m_{1}\right] \\
+ & \left.Y\left(G_{1}\right) Y\left(G_{2}\right)+12 F\left(G_{1}\right) F\left(G_{2}\right)+6 M_{1}\left(G_{1}\right) M_{1}\left(G_{2}\right)\right]-H M_{2}\left(G_{2}\right) \\
& +\left[n_{1}+10 m_{1}+10 M_{1}\left(G_{1}\right)+8 M_{2}\left(G_{1}\right)+6 F\left(G_{1}\right)+4 R e Z G_{3}\left(G_{1}\right)+Y\left(G_{1}\right)\right] \\
& +H M_{2}\left(G_{1}\right)\left[n_{2}+10 m_{2}+10 M_{1}\left(G_{2}\right)+8 M_{2}\left(G_{2}\right)+6 F\left(G_{2}\right)+4 R e Z G_{3}\left(G_{2}\right)+Y\left(G_{2}\right)\right] \\
& +Y\left(G_{2}\right)\left[m_{1}+2 M_{1}\left(G_{1}\right)+4 M_{2}\left(G_{1}\right)+F\left(G_{1}\right)+2 R e Z G_{3}\left(G_{1}\right)\right]+Y\left(G_{1}\right)\left[m_{2}+2 M_{1}\left(G_{2}\right)+4 M_{2}\left(G_{2}\right)\right. \\
& \left.F\left(G_{2}\right)+2 \operatorname{Re} Z G_{3}\left(G_{2}\right)\right]+4 R e Z G_{3}\left(G_{2}\right)\left[m_{1}+2 M_{1}\left(G_{1}\right)+2 M_{2}\left(G_{1}\right)+2 F\left(G_{1}\right)\right] . \\
& +4 R e Z G_{3}\left(G_{1}\right)\left[m_{2}+2 M_{1}\left(G_{2}\right)+2 M_{2}\left(G_{2}\right)+2 F\left(G_{2}\right)\right]+F\left(G_{2}\right)\left[3 M_{1}\left(G_{1}\right)+8 M_{2}\left(G_{1}\right)\right] \\
& +F\left(G_{1}\right)\left[3 M_{1}\left(G_{2}\right)+8 M_{2}\left(G_{2}\right)\right] .
\end{aligned}
$$


Theorem 4.3. The second hyper-Zagreb coindex of $G_{1} \otimes G_{2}$ is given by

$$
\begin{aligned}
\overline{H M}_{2}\left(G_{1} \otimes G_{2}\right)= & \frac{1}{2}\left[M_{1}\left(G_{1}\right) M_{1}\left(G_{2}\right)\right]^{2} \\
& -\frac{1}{2}\left[Y\left(G_{1}\right) Y\left(G_{2}\right)\right] \\
& -\left[2 H M_{2}\left(G_{1}\right) H M_{2}\left(G_{2}\right)\right] .
\end{aligned}
$$

Theorem 4.4. The second hyper-Zagreb coindex of $G_{1}\left[G_{2}\right]$ is given by

Proof. $\overline{H_{2}}\left(G_{1}\left[G_{2}\right]\right)=1 / 2\left[n_{2}^{3} M_{1}\left(G_{1}\right)+n_{1} M_{1}\left(G_{2}\right)+8 n_{2}\right.$ $m_{2} m_{1} Y\left(G_{1}\left[G_{2}\right]\right)=n_{2}^{5} Y\left(G_{1}\right)+n_{1} Y\left(G_{2}\right)+8 n_{2}^{3} m_{2} F\left(G_{1}\right)+8 n_{2}$ $\left.m_{1} F\left(G_{2}\right)+6 n_{2}^{2} M_{1} \quad\left(G_{1}\right) M_{1}\left(G_{2}\right)\right]^{2}+-1 / 2\left[n_{2}^{5} Y\left(G_{1}\right)+n_{1} Y\right.$ $\left.\left(G_{2}\right)+8 n_{2}^{3} m_{2} F\left(G_{1}\right)+8 n_{2} m_{1} F\left(G_{2}\right)+6 n_{2}^{2} M_{1}\left(G_{1}\right) M_{1}\left(G_{2}\right)\right]$ $-\left[n_{2}{ }^{6} H M_{2}\left(G_{1}\right)+n_{1} H M_{2} \quad\left(G_{2}\right)+n_{2}{ }^{4} m_{2}\left[Y\left(G_{1}\right)+4 \operatorname{Re} Z G_{3}\right.\right.$ $\left.\left(G_{1}\right)\right]+4 n_{2} \quad m_{1} \operatorname{Re} Z G_{3}\left(G_{2}\right)+3 n_{2}{ }^{2} F\left(G_{1}\right) M_{1}\left(G_{2}\right)+n_{2}{ }^{2} M_{1}$ $\left(G_{1}\right)\left[F\left(G_{2}\right)+4 M_{2}\left(G_{2}\right)\right]+m_{1} M_{1}^{2}\left(G_{2}\right)+4 n_{2} \quad m_{2}\left[4 n_{2} m_{2}\right.$ $\left.\left.M_{2}\left(G_{1}\right)+M_{1}\left(G_{1}\right) M_{1}\left(G_{2}\right)\right]\right]$.

In [19, 25-27], authors computed some topological indices of molecular graph of a nanotorus (Figure 4). In this section, we compute the second hyper-Zagreb coindex of a molecular graph of a nanotorus.

Corollary 4.5. Let $T=T[p ; q]$ be the molecular graph of $a$ nanotorus. Then, the first Zagreb index of a q-multiwalled nanotorus is $M_{1}\left(P_{n} \times T\right)=p q(25 n-18)$.

Proof. The proof of the above corollary is given by Gao et al. in [3]. Obviously,

$$
\begin{aligned}
\left|V\left(G_{1}\right)\right| & =\left|V\left(P_{n}\right)\right|=n, \\
\left|E\left(G_{1}\right)\right| & =\left|E\left(P_{n}\right)\right|=n-1, \\
\left|V\left(G_{2}\right)\right| & =|V(T)|=p q, \\
\left|E\left(G_{2}\right)\right| & =|E(T)|=\left(\frac{3}{2}\right) p q, \\
\left|M_{1}\left(G_{1}\right)\right| & =\left|M_{1}\left(P_{n}\right)\right|=(4 n-6), \\
\left|M_{1}\left(G_{2}\right)\right| & =\left|M_{1}(T)\right|=9 p q .
\end{aligned}
$$

Corollary 4.6. Let $T=T[p ; q]$ be the molecular graph of a nanotorus. Then, the $Y$-index of a q-multiwalled nanotorus is

$$
Y\left(P_{n} \times T\right)=p q(625 n-738) \text {. }
$$

Proof. We have by Lemma 4.2,

$$
\begin{aligned}
Y\left(G_{1} \times G_{2}\right)= & n_{2} Y\left(G_{1}\right)+n_{1} Y\left(G_{2}\right)+8 m_{1} F\left(G_{2}\right) \\
& +8 m_{2} F\left(G_{1}\right)+6 M_{1}\left(G_{1}\right) M_{1}\left(G_{2}\right) .
\end{aligned}
$$

Then,

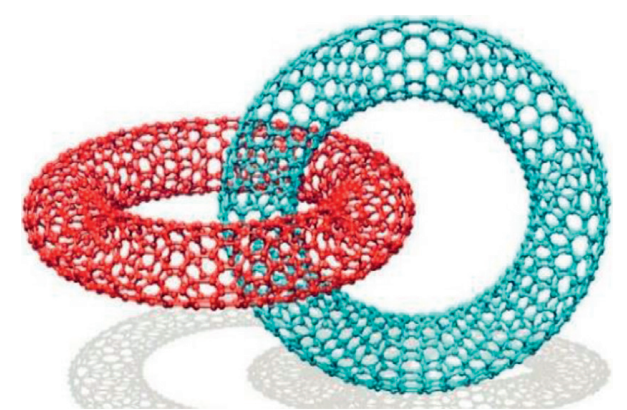

FIgURE 4: The molecular graph of a nanotorus.

$$
\begin{aligned}
Y\left(P_{n} \times T\right)= & (p q) Y\left(P_{n}\right)+n Y(T)+8(n-1) F(T) \\
& +8\left(\frac{3}{2}\right) p q F\left(P_{n}\right)+6 M_{1}\left(P_{n}\right) M_{1}(T) .
\end{aligned}
$$

Therefore,

$$
\begin{aligned}
Y\left(P_{n} \times T\right)= & (p q)(16 n-30)+n(81 p q)+8(n-1)(27 p q) \\
& +12 p q(8 n-14)+6(4 n-6)(9 p q) \\
= & p q(625 n-738) .
\end{aligned}
$$

Corollary 4.7. Let $T=T[p ; q]$ be the molecular graph of a nanotorus. Then, the second hyper-Zagreb index of a $q$ multiwalled nanotorus is

$$
H M_{2}\left(P_{n} \times T\right)=\left(\frac{1}{2}\right) p q(2561 n-3632) .
$$

Proof. We have by Lemma 2.5,

$$
\begin{aligned}
H M_{2}\left(G_{1} \times G_{2}\right)= & n_{2} H_{2}\left(G_{1}\right)+n_{1} H M_{2}\left(G_{2}\right) \\
& +3 F\left(G_{1}\right) M_{1}\left(G_{2}\right)+3 F\left(G_{2}\right) M_{1}\left(G_{1}\right) \\
& +m_{1}\left[Y\left(G_{2}\right)+4 \operatorname{ReZG}\left(G_{2}\right)\right] \\
& +m_{2}\left[Y\left(G_{1}\right)+4 \operatorname{ReZG}\left(G_{1}\right)\right] \\
& +4 M_{1}\left(G_{1}\right) M_{2}\left(G_{2}\right)+4 M_{1}\left(G_{2}\right) M_{2}\left(G_{1}\right) .
\end{aligned}
$$

As proof in Corollary 4.6, we have

$$
H M_{2}\left(P_{n} \times T\right)=\left(\frac{1}{2}\right) p q(2561 n-3632),
$$

where

$$
\left|H M_{2}\left(P_{n}\right)\right|=(16 n-40),\left|H M_{2}(T)\right|=\left(\frac{243}{2}\right) p q .
$$

Now, we apply the second hyper-Zagreb coindex on a $q$ multiwalled nanotorus using Cartesian product operation.

Corollary 4.8. Let $T=T[p ; q]$ be the molecular graph of a nanotorus. Then, the second hyper-Zagreb coindex of a $q$ multiwalled nanotorus is 


$$
\overline{H M_{2}}\left(P_{n} \times T\right)=\left(\frac{1}{2}\right) p q\left[p q(25 n-18)^{2}-3186 n+4370\right] .
$$

Proof. We have by Proposition 3.2,

$$
\overline{H M_{2}}(G)=\frac{1}{2} M_{1}^{2}(G)-\frac{1}{2} Y(G)-H M_{2}(G) .
$$

Then,

$$
\overline{H M_{2}}\left(P_{n} \times T\right)=\frac{1}{2} M_{1}^{2}\left(P_{n} \times T\right)-\frac{1}{2} Y(G)-H M_{2}\left(P_{n} \times T\right) .
$$

By using Corollaries 4.5-4.7, we obtain

$$
\overline{H M_{2}}\left(P_{n} \times T\right)=\left(\frac{1}{2}\right) p q\left[p q(25 n-18)^{2}-3186 n+4370\right] .
$$

\section{Conclusion}

In this study, we obtained the value of the second hyperZagreb coindex of some chemical graphs, and we computed some explicit formulas for their numbers under several graph operations. Also, we applied the second hyper-Zagreb coindex on a $q$-multiwalled nanotorus. The results of this work may be used as a predictor, especially in the chemical graph theory. For example, in quantitative structure-activity relationships (QSAR) modelling, the predictors consist of theoretical molecular descriptors of chemicals.

\section{Data Availability}

No data were used to support this study, except for the references that were mentioned.

\section{Conflicts of Interest}

The authors declare that they have no conflicts of interest.

\section{References}

[1] H. Ahmed, A. Alwardi, M. R. Salestina, and D. S. Nandappa, "Domination, $\gamma$-domination, topological indices and $\varphi_{p}$-polynomial of some chemical structures applied for the treatment of COVID-19 patients," Biointerface Research in Applied Chemistry, pp. 13290-13302, 2021.

[2] A. Ayache and A. Alameri, "Topological indices of the mk-graph," Journal of the Association of Arab Universities for Basic and Applied Sciences, vol. 24, no. 1, pp. 283-291, 2017.

[3] W. Gao, M. K. Siddiqui, M. Imran, M. K. Jamil, and M. R. Farahani, "Forgotten topological index of chemical structure in drugs," Saudi Pharmaceutical Journal, vol. 24, no. 3, pp. 258-264, 2016.

[4] A. Alameri, "Second hyper-zagreb index of titania nanotubes and their applications," IEEE Access, vol. 9, pp. 9567-9571, 2021.
[5] I. Gutman and N. Trinajstić, "Graph theory and molecular orbitals. Total $\varphi$-electron energy of alternant hydrocarbons," Chemical Physics Letters, vol. 17, no. 4, pp. 535-538, 1972.

[6] T. Došlić, "Vertex-weighted wiener polynomials for composite graphs," Ars Mathematica Contemporanea, vol. 1, pp. 66-80, 2008.

[7] A. R. Ashrafi, T. Došlić, and A. Hamzeh, "The Zagreb coindices of graph operations," Discrete Applied Mathematics, vol. 158, no. 15, pp. 1571-1578, 2010.

[8] G. H. Shirdel, H. Rezapour, and A. M. Sayadi, "The hyperZagreb index of graph operations," Iranian Journal of Mathematical Chemistry, vol. 4, no. 2, pp. 213-220, 2013.

[9] P. S. Ranjini, V. Lokesha, and A. Usha, "Relation between phenylene and hexagonal squeeze using harmonic index," International Journal of Graph Theory, vol. 1, no. 4, pp. 116-121, 2013.

[10] B. Furtula and I. Gutman, "A forgotten topological index," Journal of Mathematical Chemistry, vol. 53, no. 4, pp. 11841190, 2015.

[11] M. Veylaki, M. J. Nikmehr, and H. A. Tavallaee, "The third and hyper-Zagreb coindices of some graph operations," Journal of Applied Mathematics and Computing, vol. 50, no. 12, pp. 315-325, 2016.

[12] G. Wei, M. R. Farahani, and M. K. Siddiqui, "On the first and second Zagreb and first and second hyper-zagreb indices of carbon nanocones CNCk [n]," Journal of Computational and Theoretical Nanoscience, vol. 13, pp. 7475-7482, 2016.

[13] A. Alameri, N. Al-Naggar, M. Al-Rumaima, and M. Alsharafi, "Y-index of some graph operations," International Journal of Applied Engineering Research, vol. 15, no. 2, p. 179, 2020.

[14] A. Alameri, M. Al-Rumaima, and M. Almazah, "Y-coindex of graph operations and its applications of molecular descriptors," Journal of Molecular Structure, vol. 1221, Article ID 128754, 2020.

[15] U. Knauer and K. Knauer, Algebraic Graph Theory, p. p, de Gruyter, Berlin, Germany, 2019.

[16] A. Alameri, A. Modabish, and A. Ayache, "New product operation on graphs and it's properties," International Mathematical Forum, vol. 11, no. 8, pp. 357-368, 2016.

[17] Ö. Ç. Havare, "On the inverse sum indeg index of some graph operations," Journal of the Egyptian Mathematical Society, vol. 28 , no. 1, pp. 1-11, 2020.

[18] A. Modabish, A. Alameri, M. S. Gumaan, and M. Alsharafi, "The second Hyper-Zagreb index of graph operations," Journal of Mathematical and Computational Science, vol. 11, no. 2, pp. 1455-1469, 2021.

[19] I. Goldstein, A. L. Burnett, R. C. Rosen, P. W. Park, and V. J. Stecher, "The serendipitous story of sildenafil: an unexpected oral therapy for erectile dysfunction," Sexual Medicine Reviews, vol. 7, no. 1, pp. 115-128, 2019.

[20] P. Patrignani and C. Patrono, "Aspirin and cancer," Journal of the American College of Cardiology, vol. 68, no. 9, pp. 967-976, 2016.

[21] H. Ahmed, M. R. Farahani, A. Alwardi, and M. R. Salestina, "Domination topological properties of some chemical structures using $\varphi$ p-Polynomial approach," Eurasian Chemical Communications, vol. 3, no. 4, pp. 210-218, 2021.

[22] R. K. Sajja, S. Rahman, and L. Cucullo, "Drugs of abuse and blood-brain barrier endothelial dysfunction: a focus on the role of oxidative stress," Journal of Cerebral Blood Flow and Metabolism, vol. 36, no. 3, pp. 539-554, 2016.

[23] M. Ibraheem, M. M. Aljohani, M. Javaid, and A. M. Alanazi, "Forgotten coindex for the derived sum graphs under cartesian product," vol. 2021, Article ID 3235068, 13 pages, 2021. 
[24] M. Javaid, M. Ibraheem, U. Ahmad, and Q. Zhu, Computing Bounds for Second Zagreb Coindex of Sum Graphs, 2021.

[25] M. H. Khalifeh, H. Yousefi-Azari, and A. R. Ashrafi, "The first and second Zagreb indices of some graph operations," Discrete Applied Mathematics, vol. 157, no. 4, pp. 804-811, 2009.

[26] M. S. Alsharafi, M. M. Shubatah, and M. Q. Alameri, "On the Hyper-Zagreb coindex of some graphs," Journal of Mathematical and Computational Science, vol. 10, no. 5, pp. 18751890, 2020.

[27] M. Alsharafi, M. Shubatah, and A. Alameri, "The hyperZagreb index of some complement graphs," Advances in Mathematics: Scientific Journal, vol. 9, no. 6, pp. 3631-3642, 2020. 